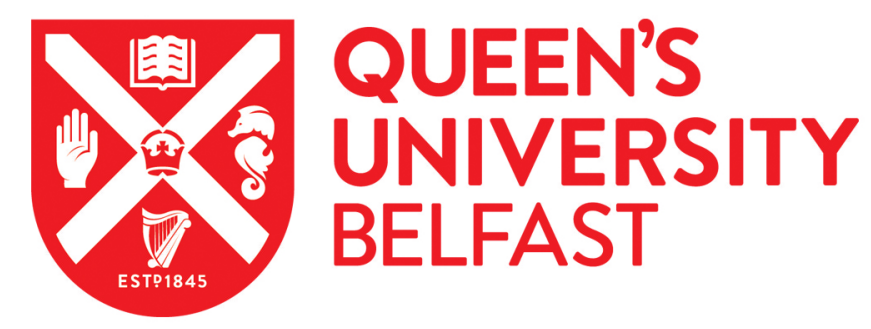

\title{
Intra-pulse transition between ion acceleration mechanisms in intense laser-foil interactions
}

Padda, H., King, M., Gray, R. J., Powell, H. W., Gonzalez-Izquierdo, B., Stockhausen, L. C., Wilson, R., Carroll, D. C., Dance, R. J., MacLellan, D. A., Yuan, X. H., Butler, N. M. H., Capdessus, R., Borghesi, M., Neely, D., \& McKenna, P. (2016). Intra-pulse transition between ion acceleration mechanisms in intense laser-foil interactions. Physics of Plasmas, 23(6), [063116]. https://doi.org/10.1063/1.4954654

Published in:

Physics of Plasmas

Document Version:

Publisher's PDF, also known as Version of record

Queen's University Belfast - Research Portal:

Link to publication record in Queen's University Belfast Research Portal

\section{Publisher rights}

(C) 2016 AIP Publishing LLC

This article may be downloaded for personal use only. Any other use requires prior permission of the author and AIP Publishing

The following article appeared in Padda, H, King, M, Gray, RJ, Powell, HW, Gonzalez-Izquierdo, B, Stockhausen, LC, Wilson, R, Carroll, DC, Dance, RJ, MacLellan, DA, Yuan, XH, Butler, NMH, Capdessus, R, Borghesi, M, Neely, D \& McKenna, P 2016, 'Intra-pulse transition between ion acceleration mechanisms in intense laser-foil interactions' Physics of Plasmas, vol 23, no. 6, 063116 and may be found at http://scitation.aip.org/content/aip/journal/pop/23/6/10.1063/1.4954654

\section{General rights}

Copyright for the publications made accessible via the Queen's University Belfast Research Portal is retained by the author(s) and / or other copyright owners and it is a condition of accessing these publications that users recognise and abide by the legal requirements associated with these rights.

Take down policy

The Research Portal is Queen's institutional repository that provides access to Queen's research output. Every effort has been made to ensure that content in the Research Portal does not infringe any person's rights, or applicable UK laws. If you discover content in the Research Portal that you believe breaches copyright or violates any law, please contact openaccess@qub.ac.uk. 


\section{Intra-pulse transition between ion acceleration mechanisms in intense laser-foil interactions}

H. Padda, M. King, R. J. Gray, H. W. Powell, B. Gonzalez-Izquierdo, L. C. Stockhausen, R. Wilson, D. C. Carroll , R. J. Dance, D. A. MacLellan, X. H. Yuan, N. M. H. Butler, R. Capdessus, M. Borghesi, D. Neely, and P. McKenna

Citation: Physics of Plasmas 23, 063116 (2016); doi: 10.1063/1.4954654

View online: http://dx.doi.org/10.1063/1.4954654

View Table of Contents: http://scitation.aip.org/content/aip/journal/pop/23/6?ver=pdfcov

Published by the AIP Publishing

\section{Articles you may be interested in}

Generation of heavy ion beams using femtosecond laser pulses in the target normal sheath acceleration and radiation pressure acceleration regimes

Phys. Plasmas 23, 063108 (2016); 10.1063/1.4953546

Enhanced laser-radiation-pressure-driven proton acceleration by moving focusing electric-fields in a foil-in-cone target

Phys. Plasmas 22, 023109 (2015); 10.1063/1.4908552

Approach towards quasi-monoenergetic laser ion acceleration with doped target

Phys. Plasmas 21, 053104 (2014); 10.1063/1.4876759

Optimized laser pulse profile for efficient radiation pressure acceleration of ions

Phys. Plasmas 19, 093112 (2012); 10.1063/1.4752214

Ultra-relativistic ion acceleration in the laser-plasma interactions

Phys. Plasmas 19, 093109 (2012); 10.1063/1.4752077

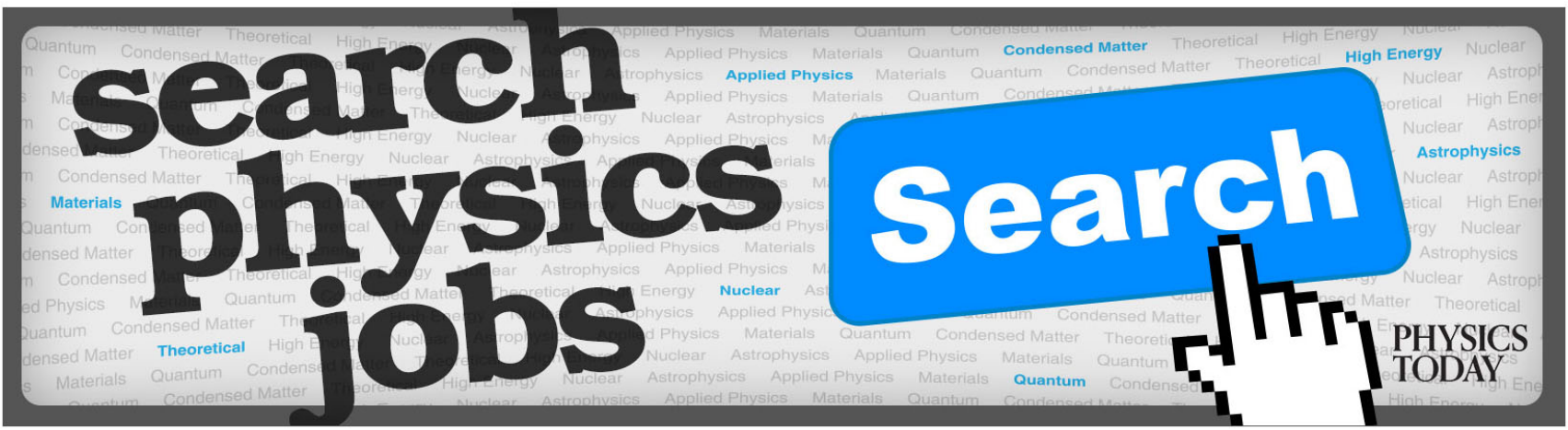




\title{
Intra-pulse transition between ion acceleration mechanisms in intense laser-foil interactions
}

\author{
H. Padda, ${ }^{1}$ M. King, ${ }^{1}$ R. J. Gray, ${ }_{1}^{1}$ H. W. Powell, ${ }^{1}$ B. Gonzalez-Izquierdo, ${ }^{1}$ \\ L. C. Stockhausen, ${ }^{2}$ R. Wilson, ${ }^{1}$ D. C. Carroll, ${ }^{3}$ R. J. Dance, ${ }^{1}$ D. A. MacLellan, ${ }^{1}$ \\ X. H. Yuan,,$^{4,5}$ N. M. H. Butler, ${ }^{1}$ R. Capdessus, ${ }^{1}$ M. Borghesi, ${ }^{6}$ D. Neely, ${ }^{1,3}$ \\ and P. McKenna ${ }^{1, a)}$ \\ ${ }^{1}$ SUPA Department of Physics, University of Strathclyde, Glasgow G4 ONG, United Kingdom \\ ${ }^{2}$ Centro de Laseres Pulsados (CLPU), Parque Cientifico, Calle del Adaja s/n. 37185 Villamayor, \\ Salamanca, Spain \\ ${ }^{3}$ Central Laser Facility, STFC Rutherford Appleton Laboratory, Oxfordshire OX11 0QX, United Kingdom \\ ${ }^{4}$ Key Laboratory for Laser Plasmas (Ministry of Education) and Department of Physics and Astronomy, \\ Shanghai Jiao Tong University, Shanghai 200240, China \\ ${ }^{5}$ Collaborative Innovation Center of IFSA (CICIFSA), Shanghai Jiao Tong University, Shanghai 200240, \\ China \\ ${ }^{6}$ Centre for Plasma Physics, Queens University Belfast, Belfast BT7 1NN, United Kingdom
}

(Received 29 April 2016; accepted 5 June 2016; published online 27 June 2016)

\begin{abstract}
Multiple ion acceleration mechanisms can occur when an ultrathin foil is irradiated with an intense laser pulse, with the dominant mechanism changing over the course of the interaction. Measurement of the spatial-intensity distribution of the beam of energetic protons is used to investigate the transition from radiation pressure acceleration to transparency-driven processes. It is shown numerically that radiation pressure drives an increased expansion of the target ions within the spatial extent of the laser focal spot, which induces a radial deflection of relatively low energy sheath-accelerated protons to form an annular distribution. Through variation of the target foil thickness, the opening angle of the ring is shown to be correlated to the point in time transparency occurs during the interaction and is maximized when it occurs at the peak of the laser intensity profile. Corresponding experimental measurements of the ring size variation with target thickness exhibit the same trends and provide insight into the intra-pulse laser-plasma evolution. Published by AIP Publishing. [http://dx.doi.org/10.1063/1.4954654]
\end{abstract}

\section{INTRODUCTION}

The acceleration of ions from thin foils irradiated by intense laser pulses offers a promising route toward the creation of compact, short pulse beams of energetic ions. ${ }^{1,2}$ Such a source may enable the development of advanced hadron therapy centers ${ }^{3-5}$ and lead to alternative approaches to inertial confinement fusion. ${ }^{6,7}$ The realization of such applications requires a deep understanding of the role of the various acceleration mechanisms that are known to occur and the development of techniques to optically control the spectral and spatial characteristics of the resultant ion beam.

Recent developments in laser and target manufacture technology have enabled experiments to be undertaken investigating laser-driven ion acceleration from nanometerthick targets. In this regime, a number of ion acceleration mechanisms have emerged as alternatives to the well-established target normal sheath acceleration (TNSA) scheme, ${ }^{8-10}$ exhibiting a faster scaling with laser intensity. Two approaches in particular, have received significant attention: the radiation pressure acceleration (RPA) $)^{11-13}$ and the transparencyenhanced sheath acceleration (or "breakout afterburner," $\mathrm{BOA}^{14}$ ) mechanisms. The onset of transparency in thin foils reduces the effectiveness of RPA, but can volumetrically heat electrons to enhance sheath fields in the BOA scheme.

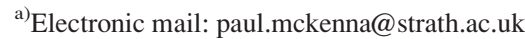

There are a number of studies in which ion energy enhancement and/or changes to the energy spectrum have been shown to be consistent with the onset of either RPA, ${ }^{15,16}$ $\mathrm{BOA},{ }^{17,18}$ or other energy transfer processes in the transparency regime. ${ }^{19}$ Time-integrated measurement of ion spectra alone is insufficient to resolve the key underlying dynamics required to determine which mechanism dominates for given target and laser pulse parameters. Moreover, recent work has shown that multiple acceleration mechanisms can occur over the duration of the laser pulse interaction with an ultrathin foil target. Signature features in the spatial-intensity distribution of the resultant ion beam, including the onset of transverse instabilities and differences in the directionality, show that TNSA, RPA, and transparency-enhanced processes can all occur at different phases of the interaction. ${ }^{20-22}$

In this article, a characterization of the intra-pulse transition from the radiation pressure-dominated to the relativistic transparency regime in ultrathin foil targets is presented. By measuring changes to the divergence of a low-energy, annular component of the proton beam, the time within the laser pulse envelope at which relativistic induced transparency (RIT) occurs can be inferred. It is shown, using particle-incell (PIC) simulations, that the proton ring is formed by RPA-driven expansion of heavier ions at the target rear, which imparts a radial force on the expanding TNSA-proton layer. The diameter of the ring is shown to be maximized when the onset of transparency occurs close to the peak of 
the pulse. Good agreement is obtained with experimental results on the scaling of the ring size with proton energy and target thickness.

\section{SIMULATION RESULTS}

To investigate the intra-pulse transition between the different ion acceleration mechanisms in ultrathin foils, 2D simulations were performed using the fully relativistic, PIC code, EPOCH. ${ }^{23}$ The simulation box was defined as $130 \mu \mathrm{m}$ $\times 72 \mu \mathrm{m}$ using $26000 \times 7200$ simulation cells with the boundaries defined as free-space. The target was initialized as a $2 \mathrm{D}$ slab of $\mathrm{Al}^{11+}$ ions with a density of $60 n_{c}$ (the density of solid aluminum) with a contamination layer of $60 n_{c}$ $\mathrm{H}^{+}$on the rear of the target, where $n_{c}=m_{e} \epsilon_{0} \omega_{L}^{2} / e^{2}\left(m_{e}\right.$ is the electron rest mass, $\epsilon_{0}$ is the vacuum permittivity, $\omega_{L}$ is the angular laser frequency, and $e$ is the electron charge). Test simulations incorporating an ionization model demonstrate that the predominant charge state achieved for $\mathrm{Al}$ is $q=11+$ for the laser parameters investigated. The electron population is defined to neutralize all of the ions appropriately with an initial temperature set to $10 \mathrm{keV}$. The thickness, $L$, of the $\mathrm{Al}^{11+}$ slab was varied in the range $L=20-500 \mathrm{~nm}$, with the contamination layer thickness kept constant at $10 \mathrm{~nm}$. The laser pulse was defined to have a Gaussian temporal profile with a full width at half maximum (FWHM) of 570 fs and was focused to a transverse Gaussian profile with a FWHM of $6 \mu \mathrm{m}$ at the front of the target. The intensity of the laser pulse was set to $2 \times 10^{20} \mathrm{~W} \mathrm{~cm}^{-2}$. To account for the laser propagation effects due to the expansion of the front surface, ${ }^{24}$ the target was positioned $30 \mu \mathrm{m}$ from the incoming laser boundary. Computationally intensive test simulations with contamination layers on both the front and rear sides, and with binary collisions enabled, show that the front surface proton layer is largely ablated and does not propagate through the $\mathrm{Al}^{11+}$ ions. With the exception of this behavior, the addition of binary collisions has negligible impact on the dynamics of the system and these were therefore not included in the simulations reported.

In all simulations it is found that early in the laser-foil interaction (i.e. at the leading edge of the laser pulse profile), electrons are accelerated from the target front side and propagate to the rear side, where they set up a strong, longitudinal sheath field, driving the TNSA mechanism. In this field, protons expand faster than the $\mathrm{Al}^{11+}$ due to their higher chargeto-mass ratio $(\mathrm{q} / \mathrm{m})$, resulting in layering of the two ion species. As the laser intensity continues to increase, the radiation pressure results in the laser pulse hole boring into the target and drives an increased longitudinal expansion of the $\mathrm{Al}^{11+}$ ions at the rear side. The maximum of this expansion occurs at the center of the laser focal spot, reducing transversely with a Gaussian profile. As the $\mathrm{Al}^{11+}$ expands into the rear of the proton layer, the electrostatic field formed at the interface between the two species begins to deflect the slowest protons toward the direction of the local normal to the $\mathrm{Al}^{11+}$ expansion profile. This results in radial proton deflection, as shown schematically in Fig. 1(a).

As the laser intensity decreases beyond the peak of the laser pulse interaction, the radiation pressure will continue to drive the transverse motion, but at a reduced rate. This behavior can be observed in Figs. 1(b) and 1(c) for an $L=500 \mathrm{~nm}$ target which does not become relativistically transparent to the laser. Figure $1(\mathrm{~b})$ shows the $\mathrm{Al}^{11+}$ and proton number density at $t=700$ fs with $t=0$ fs defined as the time when the peak of the laser pulse interacts with the front surface of the target. The Gaussian expansion profile of the $\mathrm{Al}^{11+}$ layer can be seen and by this time step the low energy proton population (in green) has been swept to either side by the induced transverse motion. Figure 1(c) shows the angular distribution of the beam of accelerated protons as a function of time. For $t<-300 \mathrm{fs}$, TNSA dominates and there is a divergent beam with no observed splitting. At approximately $t=-300$ fs the radiation pressure is sufficient that the expansion of the $\mathrm{Al}$ ions starts deflecting the low energy protons to larger angles. The width of the resulting annular profile, $\Delta \theta$ (effectively the ring diameter in 3D), increases throughout the remainder of the interaction. The target thickness is such that it remains opaque to the laser light. A ring is not produced at higher proton energies (blue in Figs. 1(c) and 1(d)).

For a sufficiently thin target, heating and expansion of the electron population will result in it becoming relativistically transparent during the laser pulse interaction. As an example, Figs. 1(d) and 1(e) show the case for $L=40 \mathrm{~nm}$, for which RIT occurs at $t=20 \mathrm{fs}$. As with the thicker target, the relatively low energy proton beam component starts to undergo radial deflection at approximately $t=-300 \mathrm{fs}$. However, the overall rate of increase in $\Delta \theta$ is larger due to the increased velocity of expansion of the Al ions. Thus, the diameter of the final proton ring depends on whether RIT occurs and, as will be shown below, on when it occurs with respect to the peak of the laser pulse profile.

Two further observations are worthy of note: (1) The overall target expansion profile is similar to that previously observed experimentally in intense laser pulse interactions with thin foil targets; ${ }^{25}$ (2) A jet of high energy ions can also be observed propagating close to the $\mathrm{Y}=0$ axis in Fig. 1(d). This is a feature of the transparency-enhanced acceleration regime, as previously reported in Powell et al. ${ }^{20}$

In Fig. 2 the temporal evolution of $\Delta \theta$ is shown for given $L$ in the range of $20-500 \mathrm{~nm}$, along with the idealized temporal profile of the laser intensity envelope arriving at the target. In all cases the proton beam splits at around $t=-300 \mathrm{fs}$, this occurs slightly earlier for small $L$ and later for large $L$. As the intensity continues to increase, $\Delta \theta$ increases for all $L$, but the rate of change differs. The rate is generally higher for small $L$, within the RPA-dominated phase of the interaction. However, if RIT occurs early in the interaction then the final ring beam diameter is smaller than if it occurs near the peak of the laser profile. This is clearly observed in Fig. 2 when comparing the $L=20 \mathrm{~nm}$ and $L=40 \mathrm{~nm}$ cases (where the dotted vertical line marks the time at which RIT occurs for each $L$ ). A comparison with the $L=100 \mathrm{~nm}$ case, for which RIT occurs on the falling edge of the laser pulse, shows that the largest ring is obtained when RIT occurs near the peak of the laser intensity, at which the hole-boring velocity is highest. 

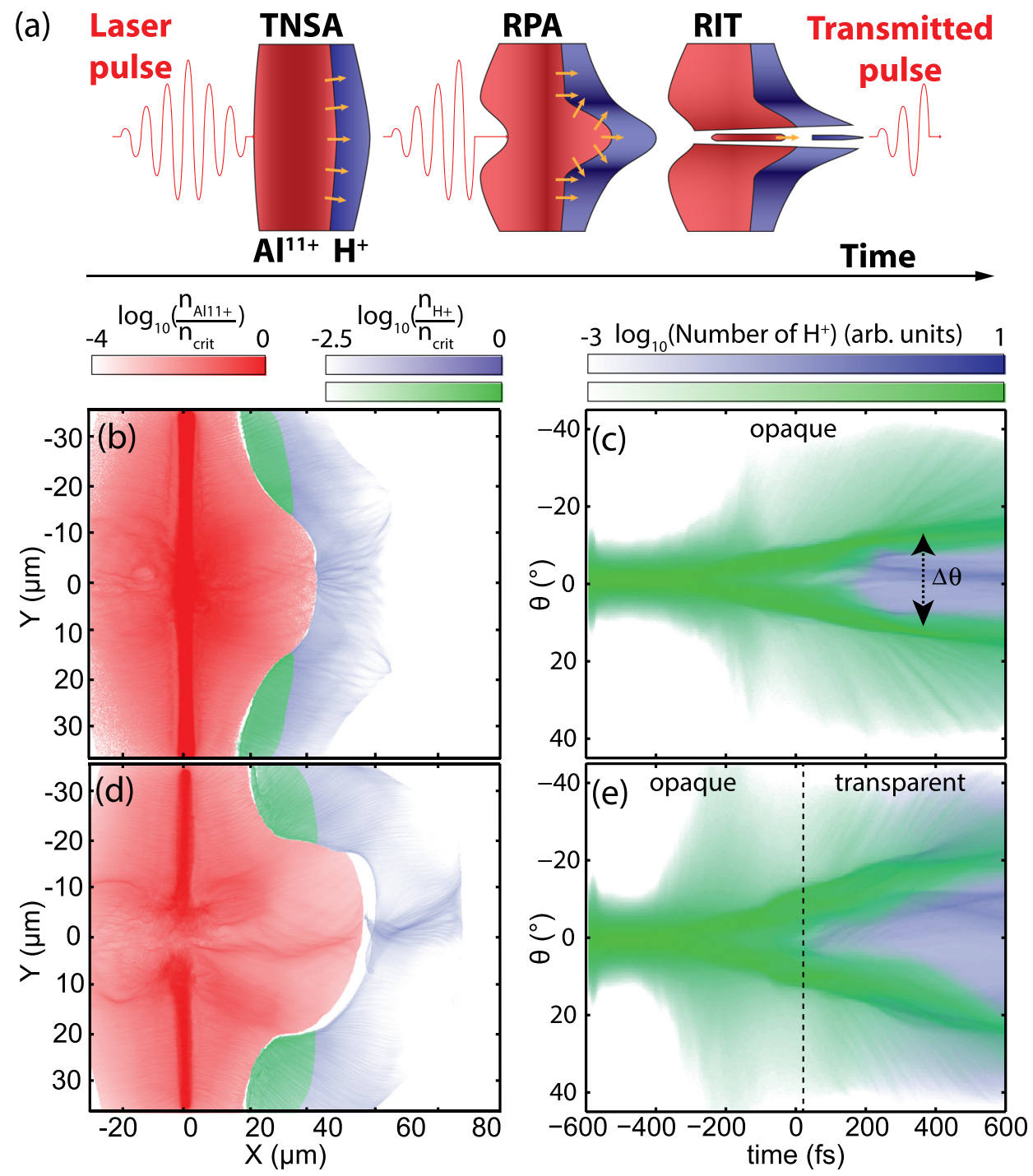

FIG. 1. (a) Schematic illustrating the three stages of ion acceleration: TNSA driven by energetic electrons early in the interaction; a hole-boring-RPA phase in which $\mathrm{Al}$ ions are accelerated into the back of the expanding proton layer, giving rise to radial expulsion; the onset of RIT. (b) and (c) Example simulation results showing: (b) Ion densities for an $L=500 \mathrm{~nm}$ target at $t=700 \mathrm{fs}$ after the interaction of the peak of the laser pulse: Red$\mathrm{Al}^{11+}$ ions; Green-protons with energy in the lower quartile; Blue-remainder, higher energy protons. (c) Angular profile of the protons accelerated from the $L=500 \mathrm{~nm}$ target as a function of time with respect to the peak of the pulse $(t=0)$. (d) and (e) Same for $L=40 \mathrm{~nm}$, with same scales.

\section{EXPERIMENT RESULTS}

To test the physical picture emerging from the simulation results, an experimental study was performed using the $1.054 \mu \mathrm{m}$ wavelength Vulcan laser at the Rutherford Appleton Laboratory. This laser delivered pulses of $(0.8 \pm 0.2)$ ps FWHM duration focused to a spot diameter of $8 \mu \mathrm{m}$ FWHM. A single plasma mirror was employed to increase the intensity contrast from $10^{8}$ to $\sim 10^{10}$ at $\sim 40$ ps prior to the peak of the pulse..$^{20}$ This resulted in an on-target laser pulse energy of (200 \pm 25$) \mathrm{J}$, giving a calculated peak intensity, $I_{L}=2 \times 10^{20} \mathrm{~W} / \mathrm{cm}^{2}$. The laser was linearly polarized and was aligned at near-normal incidence to $\mathrm{Al}$ foil targets with a thickness, $L$, varied between $10 \mathrm{~nm}$ and $400 \mathrm{~nm}$.

The measurement of the spatial-intensity distribution of the beam of accelerated protons was achieved using a stack of dosimetry (radiochromic, RCF) film with dimensions of $6.5 \mathrm{~cm} \times 5.0 \mathrm{~cm}$. This enabled the spatial distribution to be measured in discrete energy bands for $E_{\text {prot }}$ ranging from 2.7 to $45 \mathrm{MeV}$. A horizontal slot was cut through the center of the stack in order to provide a line-ofsight to additional diagnostics and the stack was positioned $6 \mathrm{~cm}$ from the rear of the target. A thin PTFE film was also positioned at the front of the stack and the diffuse light generated by the transmitted laser light was imaged using a CCD camera.

An annular beam profile was observed for low energy protons, as shown in the representative measurements of the spatial-intensity profile in Figs. 3(a) and 3(b) and corresponding dose profiles along the vertical axis shown in Figs. $3(\mathrm{c})$ and $3(\mathrm{~d})$. For fixed $L=10 \mathrm{~nm}, \Delta \theta$ of the inner part of the ring can be seen to increase with $E_{\text {prot }}$, as shown in Figs. 3(a) and 3(c). For higher $E_{\text {prot }}$, the annular structure becomes undetectable, resulting in a low divergent, high energy component as seen in prior studies. ${ }^{20,26}$ In Figs. 3(b) and 3(d), $\Delta \theta$ is also observed to vary with $L$, and is largest for $L=80 \mathrm{~nm}$. For thinner targets radial instabilities (manifested in spoke structures) can also be observed and may be associated with RIT effects. A more detailed investigation of these instabilities is outside the scope of this article and will be the subject of follow-on work.

Figure 4 compares the quantitative results from the experiment and simulations. As observed in Fig. 4(a), both exhibit an optimal target thickness, $L_{o p t}$, which produces the largest divergence angle in the low-energy proton ring. The difference in the absolute value $\left(L_{\text {opt }}=80 \mathrm{~nm}\right.$ in the experiment and $40 \mathrm{~nm}$ in the simulations) is attributed to 


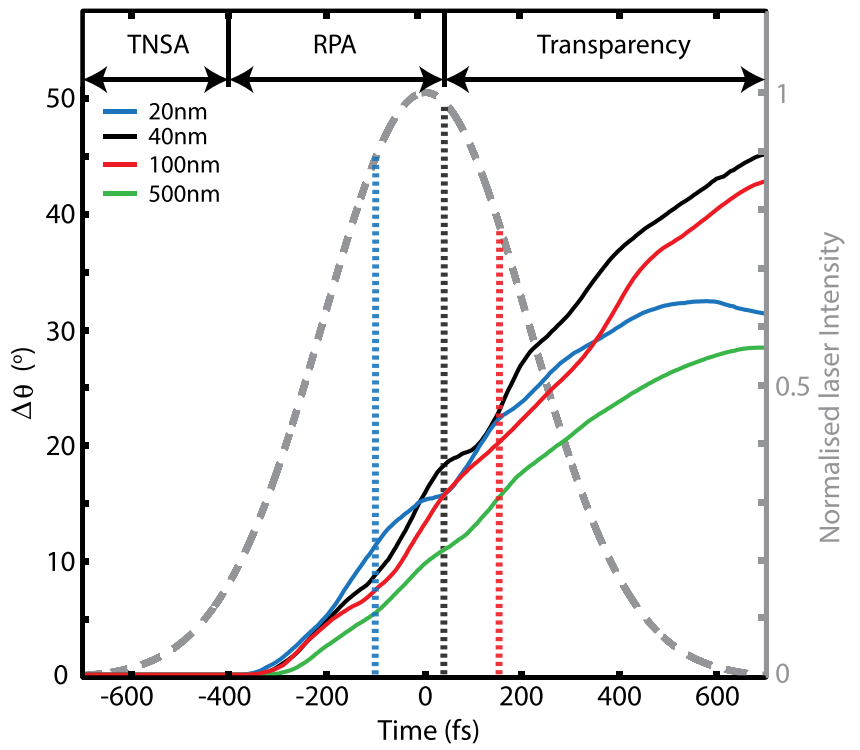

FIG. 2. Simulation results showing the temporal behavior of the average ring divergence angle for given target thicknesses. The temporal profile of the laser intensity is also shown with dashed vertical lines added to indicate the onset of transparency for the corresponding target thickness. Note that the $L=500 \mathrm{~nm}$ target does not undergo transparency. The dominant intrapulse acceleration mechanisms are labeled at the top of the figure for the $L=40 \mathrm{~nm}$ example case.

the idealized parameters and 2D dimensionality of the simulations. A comparison with Fig. 2 reveals that $L_{\text {opt }}$ corresponds to the scenario in which RIT occurs at (or close to) the peak of the laser pulse profile. When the target thickness in the simulation results is scaled up by a factor of two to take into account of this, good agreement is observed with the experiment results over most of the thickness range. For $L \geq 400 \mathrm{~nm}$ the simulations continue to show a transverse deflection of the lowest energy protons, whereas the ring is not observed experimentally. It should be noted though, that the maximum measured proton energy decreases with increasing $L$, and as the ring is only produced in the low energy proton population, it is possible that it exists at energies below the lower detection threshold (equal to $2 \mathrm{MeV}$ ) of the dosimetry film stack. Otherwise, the overall measured scaling of the ring size with target thickness is similar to that predicted in the simulations.

Figure 4(b) presents $\Delta \theta$ as a function of $E_{\text {prot }}$, normalized to the maximum proton energy $\left(E_{\max }\right)$ of the detected annular component. This is shown experimentally for $L=10-80 \mathrm{~nm}$ and compared with the simulations for $L=20-100 \mathrm{~nm}$. The energy dependence of $\Delta \theta$ follows a similar trend in both cases. For $L<L_{\text {opt }}$, the increase in $\Delta \theta$ with $E_{\text {prot }}$ is much greater than for $L \geq L_{\text {opt }}$, which further highlights the change in behavior when $L=L_{\text {opt }}$.

Figure 4(c) displays the measured transmitted light as a function of $L$, alongside the laser energy transmitted in the simulations. The percentage of laser light transmitted is observed to decrease with increasing $L$, as expected. For $L \geq L_{\text {opt }}$ (where $\Delta \theta$ varies little with proton energy), the percentage of transmitted light is low. It increases rapidly with decreasing $L$ for $L<L_{\text {opt }}$. Thus the onset of RIT is shown to change the ion expansion dynamics, and thereby the proton ring diameter, and how this varies with proton energy. (a)

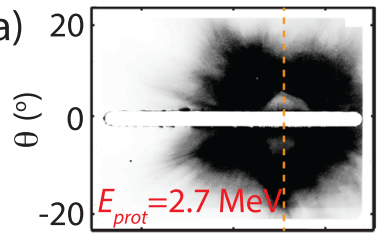

(b)

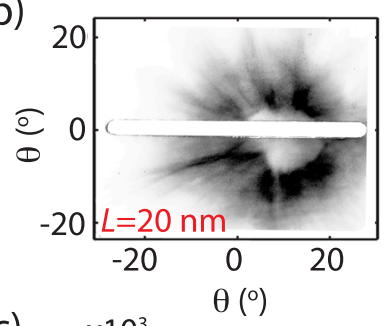

(c)

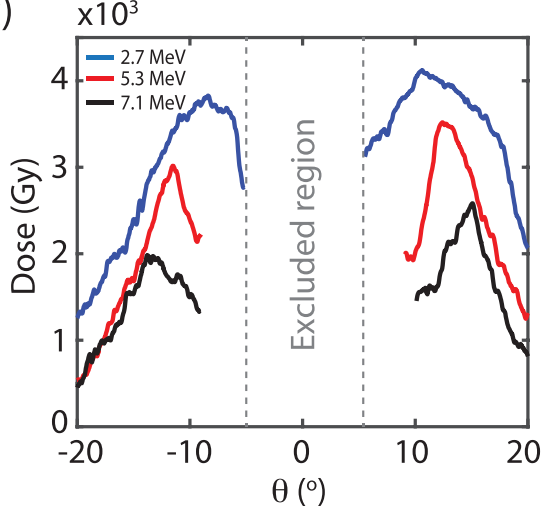

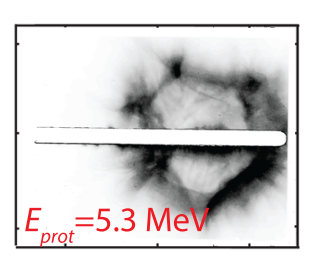
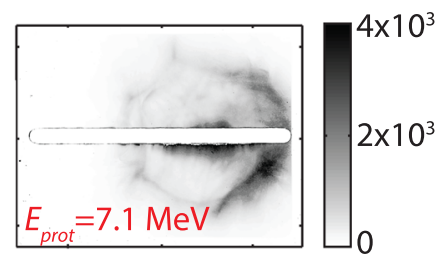

\section{恿}
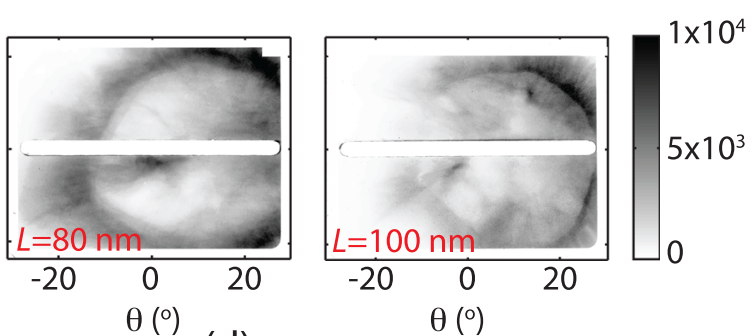

(d) 1.5

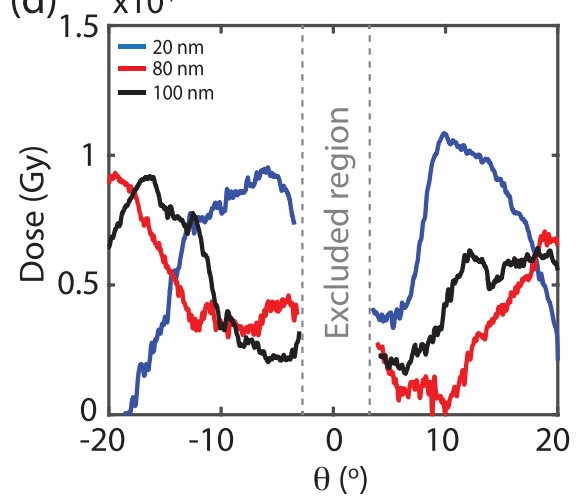

FIG. 3. Measured proton spatialintensity dose profiles for: (a) Given proton energies $\left(E_{\text {prot }}=2.7-7.1 \mathrm{MeV}\right)$ for $L=10 \mathrm{~nm}$; (b) Given $L$ for $E_{\text {prot }}=2.7 \mathrm{MeV}$; (c) Vertical line-outs through (a); (d) Vertical line-outs through (b). The angular range missing in (c) and (d) is due to a slot in the RCF. 
(a)

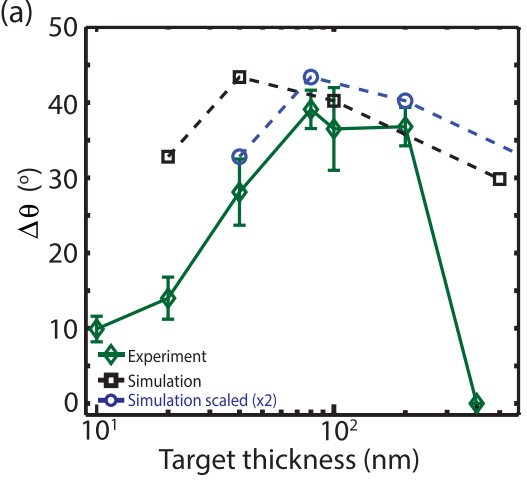

(b)

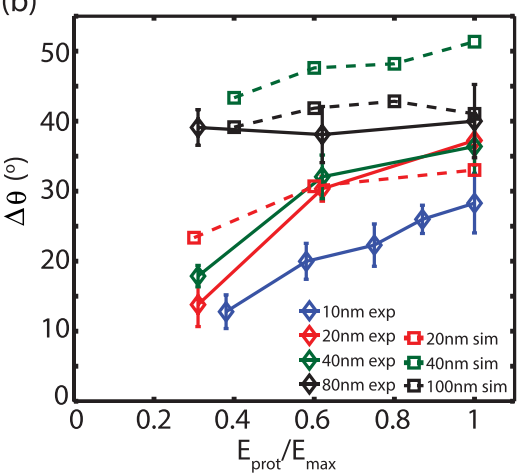

(c)

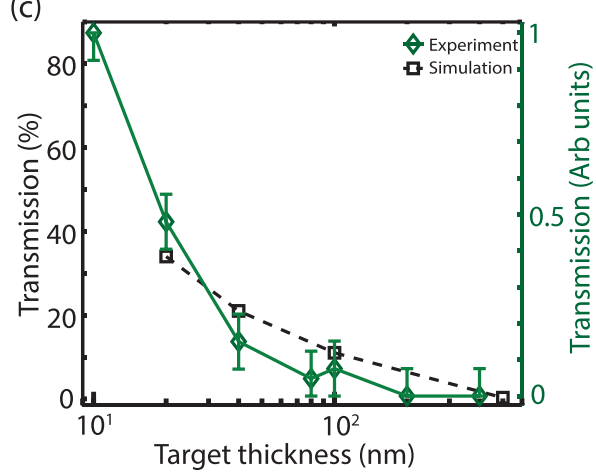

FIG. 4. Comparison of experiment and simulation results. (a) $\Delta \theta$ as a function of $L$, for low energy protons ( $E_{\text {prot }}=2.7 \mathrm{MeV}$ in the experiment and integrated over the lower $20 \%$ of the proton energy range in the simulations). The simulation results scaled by a factor of two in target thickness (as determined by the difference in $L_{o p t}$ ) is also shown. (b) Divergence angle $\Delta \theta$ as a function of normalized proton energy. (c) Transmitted laser light as a function of $L$.

\section{CONCLUSION}

In conclusion, analysis of the angular emission of the low energy component of the beam of accelerated protons provides new insight into ultrathin target dynamics during ion acceleration. In particular, monitoring how the annular low energy components vary as a function of target thickness can be used to identify the transition between RPA and transparency enhanced charged particle dynamics, and to select the appropriate targets for investigating either mechanism. This approach can be combined with measurements of the laser transmission, and possibly the duration of the transmitted pulse, to provide new insight into the intra-pulse interaction dynamics, advancing the development of laser-driven ion-acceleration.

\section{ACKNOWLEDGMENTS}

We acknowledge the support of Central Laser Facility staff and the use of the ARCHIE-WeST and ARCHER computers. This work was supported by EPSRC (Grant Nos. EP/J003832/1, EP/L001357/1, EP/K022415/1, EP/M018091/1, and EP/L000237/1), STFC (Grant No. ST/K502340/1), the U.S. Air Force Office of Scientific Research (Grant No. FA8655-13-1-3008), and the European Unions Horizon 2020 research and innovation program (Grant Agreement No. 654148 Laserlab-Europe). L.C.S. acknowledges the EU-funded LA3NET consortium (Grant No. GA-ITN-2011-289191) and X.H.Y. acknowledges support from the National Basic Research Program of China (Grant No. 2013CBA01502). EPOCH was developed under EPSRC Grant No. EP/G054940/ 1. Data associated with the research published in this paper are accessible at http://dx.doi.org/10.15129/78a76f3e-e997-497c$8 \mathrm{c} 87-6 \mathrm{e} 2 \mathrm{c} 4 \mathrm{c} 019109$.

${ }^{1}$ H. Daido, M. Nishiuchi, and A. S. Pirozhkov, Rep. Prog. Phys. 75, 056401 (2012).

${ }^{2}$ A. Macchi, M. Borghesi, and M. Passoni, Rev. Mod. Phys. 85, 751 (2013).

${ }^{3}$ S. V. Bulanov, T. ZH. Esirkepov, V. S. Khoroshkov, A. V. Kuznetsov, and F. Pegoraro, Phys. Lett. A 299, 240 (2002).

${ }^{4}$ E. Fourkal, B. Shahine, M. Ding, J. S. Li, T. Tajima, and C.-M. Ma, Med. Phys. 29, 2788 (2002).

${ }^{5}$ V. Malka, S. Fritzler, E. Lefebvre, E. d'Humieres, R. Ferrand, G. Grillion, C. Albaret, S. Meyroneinc, J. Chambaretm, A. Antonetti, and D. Hulin, Med. Phys. 31, 1587 (2004).
${ }^{6}$ M. Roth, T. E. Cowan, M. H. Key, S. P. Hatchett, C. Brown, W. Fountain, J. Johnson, D. M. Pennington, R. A. Snavely, S. C. Wilks, K. Yasuike, H. Ruhl, F. Pegoraro, S. V. Bulanov, E. M. Campbell, M. D. Perry, and H. Powell, Phys. Rev. Lett. 86, 436 (2001).

${ }^{7}$ M. Temporal, J. J. Honrubia, and S. Atzeni, Phys. Plasma. 15, 052702 (2008).

${ }^{8}$ E. L. Clark, K. Krushelnick, J. R. Davies, M. Zepf, M. Tatarakis, F. N. Beg, A. Machacek, P. A. Norreys, M. I. K. Santala, I. Watts, and A. E. Dangor, Phys. Rev. Lett. 84, 670 (2000).

${ }^{9}$ A. Maksimchuk, S. Gu, K. Flippo, D. Umstadter, and V. Yu. Bychenkov, Phys. Rev. Lett. 84, 4108 (2000).

${ }^{10}$ R. A. Snavely, M. H. Key, S. P. Hatchett, T. E. Cowan, M. Roth, T. W. Phillips, M. A. Stoyer, E. A. Henry, T. C. Sangster, M. S. Singh, S. C. Wilks, A. MacKinnon, A. Offenberger, D. M. Pennington, K. Yasuike, A. B. Langdon, B. F. Lasinski, J. Johnson, M. D. Perry, and E. M. Campbell, Phys. Rev. Lett. 85, 2945 (2000).

${ }^{11}$ T. Esirkepov, M. Borghesi, S. V. Bulanov, G. Mourou, and T. Tajima, Phys. Rev. Lett. 92, 175003 (2004).

${ }^{12}$ A. Macchi, S. Veghini, and F. Pegoraro, Phys. Rev. Lett. 103, 085003 (2009).

${ }^{13}$ A. Henig, S. Steinke, M. Schnürer, T. Sokollik, R. Hörlein, D. Kiefer, D. Jung, J. Schreiber, B. M. Hegelich, X. Q. Yan, J. Meyer-ter-Vehn, T. Tajima, P. V. Nickles, W. Sandner, and D. Habs, Phys. Rev. Lett. 103, 245003 (2009).

${ }^{14}$ L. Yin, B. J. Albright, B. M. Hegelich, and J. C. Fernandez, Laser Part. Beams 24, 291 (2006).

${ }^{15}$ A. Macchi, S. Veghini, T. V. Liseykina, and F. Pegoraro, New J. Phys. 12, 045013 (2010).

${ }^{16}$ S. Kar, K. F. Kakolee, B. Qiao, A. Macchi, M. Cerchez, D. Doria, M. Geissler, P. McKenna, D. Neely, J. Osterholz, R. Prasad, K. Quinn, B. Ramakrishna, G. Sarri, O. Willi, X. Y. Yuan, M. Zepf, and M. Borghesi, Phys. Rev. Lett. 109, 185006 (2012).

${ }^{17}$ L. Yin, B. J. Albright, B. M. Hegelich, K. J. Bowers, K. A. Flippo, T. J. T. Kwan, and J. C. Fernández, Phys. Plasma 14, 056706 (2007).

${ }^{18}$ D. Jung, B. J. Albright, L. Yin, D. C. Gautier, R. Shah, S. Palaniyappan, S. Letzring, B. Dromey, H. C. Wu, and T. Shimada, New J. Phys. 15, 123035 (2013).

${ }^{19}$ S. Palaniyappan, C. Huang, D. C. Gautier, C. E. Hamilton, M. A. Santiago, C. Kreuzer, A. B. Sefkow, R. C. Shah, and J. C. Fernández, Nat. Commun. 6, 10170 (2015).

${ }^{20}$ H. W. Powell, M. King, R. J. Gray, D. A. MacLellan, B. GonzalezIzquierdo, L. C. Stockhausen, G. Hicks, N. P. Dover, D. R. Rusby, D. C. Carroll, H. Padda, R. Torres, S. Kar, R. J. Clarke, I. O. Musgrave, Z. Najmudin, M. Borghesi, D. Neely, and P. McKenna, New J. Phys. 17, 103033 (2015).

${ }^{21}$ F. Wagner, S. Bedacht, V. Bagnoud, O. Deppert, S. Geschwind, R. Jaeger, A. Ortner, A. Tebartz, B. Zielbauer, D. H. H. Hoffmann, and M. Roth, Phys. Plasma 22, 063110 (2015).

${ }^{22}$ B. Gonzalez-Izquierdo, R. J. Gray, M. King, R. J. Dance, R. Wilson, J. McCreadie, N. M. H. Butler, R. Capdessus, S. Hawkes, J. S. Green, M. Borghesi, D. Neely, and P. McKenna, Nat. Phys. 12, 505 (2016).

${ }^{23}$ T. D. Arber, K. Bennett, C. S. Brady, A. Lawrence-Douglas, M. G. Ramsay, N. J. Sircombe, P. Gillies, R. G. Evans, H. Schmitz, A. R. Bell, and C. P. Ridgers, Plasma Phys. Controlled Fusion 57, 113001 (2015). 
${ }^{24}$ R. J. Gray, D. C. Carroll, X. H. Yuan, C. M. Brenner, M. Burza, M. Coury, K. L. Lancaster, X. X. Lin, Y. T. Li, D. Neely, M. N. Quinn, O. Tresca, C.-G. Wahlström, and P. McKenna, New J. Phys. 16, 113075 (2014).

${ }^{25}$ S. Kar, M. Borghesi, S. V. Bulanov, M. H. Key, T. V. Liseykina, A. Macchi, A. J. Mackinnon, P. K. Patel, L. Romagnani, A. Schiavi, and O. Willi, Phys. Rev. Lett. 100, 225004 (2008).
${ }^{26}$ N. Dover, C. A. J. Palmer, M. J. V. Streeter, H. Ahmed, B. Albertazzi, M. Borghesi, D. C. Carroll, J. Fuchs, R. Heathcote, P. Hilz, K. F. Kakolee, S. Kar, R. Kodama, A. Kon, D. A. MacLellan, P. McKenna, S. R. Nagel, D. Neely, M. M. Notley, M. Nakatsutsumi, R. Prasad, G. Scott, M. Tampo, M. Zepf, J. Schreiber, and Z. Najmudin, New J. Phys. 18, 013038 (2016). 